\title{
Stratogenèse associée à la dynamique des coulées à front pierreux en milieu alpin, La Mortice, Alpes méridionales, France
}

\section{Bedding Associated to the Dynamics of Stone-Banked Sheets in an Alpine Environment (La Mortice, Southern Alpes, France) Stratogenese in Verbindung mit der Dynamik von Strömungen mit steiniger Front im Alpenmilieu, La Mortice, südliche Alpen, Frankreich}

\section{Pascal Bertran, Bernard Francou et Pierre Pech}

Volume 47, numéro 1, 1993

URI : https://id.erudit.org/iderudit/032933ar

DOI : https://doi.org/10.7202/032933ar

\section{Aller au sommaire du numéro}

Éditeur(s)

Les Presses de l'Université de Montréal

ISSN

0705-7199 (imprimé)

1492-143X (numérique)

Découvrir la revue

Citer cet article

Bertran, P., Francou, B. \& Pech, P. (1993). Stratogenèse associée à la dynamique des coulées à front pierreux en milieu alpin, La Mortice, Alpes méridionales, France. Géographie physique et Quaternaire, 47(1), 93-100.

https://doi.org/10.7202/032933ar
Résumé de l'article

L'étude des dépôts de pente nappant les versants est et nord-ouest de La Mortice (3170 m, Alpes méridionales, France) met en évidence le rôle de la dynamique des coulées de solifluxion à front pierreux dans le développement d'une stratification. Au sein des coulées, la cryoexpulsion et le lavage produisent la différenciation d'un front caillouteux associé à un pavage superficiel et d'un niveau riche en matrice. Ce dernier progresse par cryoreptation et ensevelit le front pierreux au fur et à mesure de sa constitution, pour former ainsi un lit grossier enterré à structure ouverte. La cryoturbation et des transferts de matière hors du système constitué par la coulée (éluviation de la matrice, expulsion et transit des blocs en surface) s'opèrent pendant le dépôt et perturbent la stratogénèse. La comparaison des dépôts provenant des deux versants de La Mortice ainsi que des exemples déjà connus dans les Andes montre que la stratification est d'autant mieux exprimée que le nombre de cycles gel-dégel annuels est important et que la profondeur d'engel est faible.
Tous droits réservés ㄷ Les Presses de l'Université de Montréal, 1993

Ce document est protégé par la loi sur le droit d'auteur. L'utilisation des services d'Érudit (y compris la reproduction) est assujettie à sa politique d'utilisation que vous pouvez consulter en ligne.

https://apropos.erudit.org/fr/usagers/politique-dutilisation/ 


\section{STRATOGENĖSE ASSOCIÉE À LA DYNAMIQUE DES COULÉES À FRONT PIERREUX EN MILIEU ALPIN, LA MORTICE, ALPES MÉRIDIONALES, FRANCE}

Pascal BERTRAN, Bernard FRANCOU et Pierre PECH, respectivement: Institut du Quaternaire, Bâtiment de géologie, Avenue des Facultés, 33405 Talence, France; Centre de géomorphologie du CNRS, Rue des Tilleuls, 14000 Caen, France; U.F.R. de géographie, Université de Paris I, 191, rue Saint-Jacques, 75005 Paris, France.

RÉSUMÉ L'étude des dépôts de pente nappant les versants est et nord-ouest de La Mortice (3170 m, Alpes méridionales, France) met en évidence le rôle de la dynamique des coulées de solifluxion à front pierreux dans le développement d'une stratification. Au sein des coulées, la cryoexpulsion et le lavage produisent la différenciation d'un front caillouteux associé à un pavage superficiel et d'un niveau riche en matrice. Ce dernier progresse par cryoreptation et ensevelit le front pierreux au fur et à mesure de sa constitution, pour former ainsi un lit grossier enterré à structure ouverte. La cryoturbation et des transferts de matière hors du système constitué par la coulée (éluviation de la matrice, expulsion et transit des blocs en surface) s'opèrent pendant le dépôt et perturbent la stratogénèse. La comparaison des dépôts provenant des deux versants de La Mortice ainsi que des exemples déjà connus dans les Andes montre que la stratification est d'autant mieux exprimée que le nombre de cycles gel-dégel annuels est important et que la profondeur d'engel est faible.
ABSTRACT Bedding associated to the dynamics of stone-banked sheets in an alpine environment (La Mortice, southern Alpes, France). This paper emphasizes the role of stone-banked sheets in bedding of slope deposits at La Mortice $(3170 \mathrm{~m})$. Coarse particles sorting due to frost heaving and runoff occurs within sheets leading to the development of stone banks and surface pavements in connection with matrix-rich beds. Burying of stones at the sheet front by the frost creep induced movement of matrixrich layers makes it possible the formation of coarse openwork beds. Cryoturbation, heaving of blocky materials and intense leaching of fine particles through the different beds disturb the stratification. A comparison between deposits from the east and the northwest facing slopes of La Mortice and Andean examples clearly shows that bedding is better developped when superficial freeze-thaw cycles are frequent and the depth of seasonal freezing shallow.
ZUSAMMENFASSUNG Stratogenese in Verbindung mit der Dynamik von Ström. ungen mit steiniger Front im Alpenmilieu, La Mortice, südliche Alpen, Frankreich. Das Studium der Hangablagerungen, welche die östlichen und nordwestlichen Hänge von La Mortice überziehen ( 3170 m, südliche Alpen, Frankreich), hebt deutlich die Rolle der Dynamik des Bodenfließens mit steiniger Front bei der Entwicklung einer Schichtung hervor. Innerhalb der Strömungen bewirken Frostauswerfung und Auswaschung die Differenzierung einer kiesartigen Front verbunden mit einer oberflächlichen Bedeckung und einem an Grundmasse reichen Niveau. Letzteres bewegt sich durch Frostkriechen und beerdigt im Laufe seiner Entstehung die steinige Front, bis es ein grobes eingegrabenes Bett mit offener Struktur bildet. Kryoturbation und Materialtransfer außerhalb des durch die Strömung gebildeten Systems (Verwitterung der Grundmasse, Aussto $ß$ und Transit der Oberflächenblöcke) finden während der Ablagerung statt und stören die Stratogenese. Der Vergleich der Ablagerung von den zwei Hängen von La Mortice sowie von schon bekannten Beispielen in den Anden zeigt, daß die Schichtung umso besser ausgedrückt ist, je bedeutender die Zahl der jährlichen Frost- und Tauperioden und je schwächer die Frosttiefe ist. 


\section{INTRODUCTION}

Le rôle des coulées et nappes-coulées à front pierreux dans la genèse des formations de pente stratifiées en milieu périglaciaire a été démontré dans les Andes (Francou, 1988, 1990), en contexte subéquatorial à cycles de gel-dégel fréquents et superficiels associés à un manteau neigeux éphémère. La mise en évidence de ces mécanismes de stratogénèse a conduit à réexaminer des dépôts stratifiés hérités du type grèzes litées en Europe sud-occidentale. Leur étude a fait apparaître de nombreuses similitudes aux différentes échelles d'observation avec les formations étudiées dans les Andes, supposant ainsi une identité de mise en place (Francou, 1990; Bertran et al., 1992). Néanmoins, l'attribution d'une signification paléoclimatique précise à ces dépôts se heurte à la rareté des observations concernant la présence et l'organisation détaillée des formations stratifiées actives dans les milieux périglaciaires situés hors des tropiques.

En effet, des dépôts lités actifs n'ont été que rarement signalés sous les moyennes et les hautes latitudes, dans un contexte de gel et de couverture neigeuse saisonniers (Malaurie, 1968; Jahn, 1970 in Van Vliet-Lanoë, 1989; Soutadé, 1975; Vergès, 1982, Van Vliet-Lanoë, 1987). ॥ apparaît donc d'un intérêt certain d'étudier les mécanismes de ségrégation granulométrique intervenant sur les coulées à front pierreux dans ces régions et de vérifier dans quelle mesure ils donnent naissance à des litages comparables à ceux observés à la fois dans les hautes montagnes équatoriales et dans le domaine européen fossile.

Cette question constitue l'objet de cet article. II s'agit d'une analyse effectuée sur le sommet de La Mortice, dans les Alpes françaises méridionales, site déjà connu pour avoir suscité des recherches en géocryologie (Coutard et al., 1988; Van Vliet-Lanoë, 1988a et b) et donc bien documenté.

\section{ENVIRONNEMENT PÉRIGLACIAIRE DU SITE}

Le sommet de La Mortice $\left(44^{\circ} 35^{\prime} \mathrm{N}, 6^{\circ} 46^{\prime} \mathrm{E}\right)$ est situé dans les Alpes de Haute-Provence (fig. 1), au nord du col de Vars, entre 3050 et $3170 \mathrm{~m}$ d'altitude, soit approximativement à la hauteur de l'isotherme $-3^{\circ} \mathrm{C}$ de température moyenne annuelle de l'air (isotherme $0^{\circ} \mathrm{C}$ à $2500 \mathrm{~m}$ ). Les enregistrements thermiques montrent la présence d'un gélisol d'octobre à juin-juillet, avec un front de gel pénétrant dans le sol à une profondeur supérieure à $100 \mathrm{~cm}$. Le nombre de cycles de gel-dégel annuels en surface oscille entre 40 en zone de fort enneigement et 130 en zone de faible enneigement. $\dot{A}$ partir de $30 \mathrm{~cm}$ de profondeur, il ne se produit plus qu'un seul cycle annuel (J.-P. Coutard, comm. orale). L'existence d'un pergélisol lacunaire sur le site est probable, mais devra attendre confirmation après dépouillement complet des données thermiques.

La roche mère est un calcschiste (flysch noir) d'âge paléocène reposant sur une dalle calcaire karstifiée du Jurassique supérieur. Ce matériel produit par gélifraction une grande quantité de débris inférieurs à $2 \mathrm{~mm}$, dont 6 à $16 \%$ d'argile.

La dynamique de versant est très active et dominée par la cryoreptation (Coutard et al., 1988; Van Vliet-Lanoë, 1988a).
Ce processus est responsable d'un déplacement moyen de $2 \mathrm{~cm}$.an-1 des fragments à la surface du sol. La gélifluxion et les déplacements dans le domaine plastique-liquide interviennent à certaines périodes de l'année et d'une façon localisée dans le transport des matériaux: fonte des neiges au printemps sur sol gelé, averses concentrées pendant l'été, qui sont à l'origine de petites laves boueuses (debris flows). En surface, les figures de tri sont fréquentes (sols striés, polygones, ostioles) et présentent généralement un maillage métrique.

Deux versants à morphologie et pédoclimat différents ont été étudiés. Le premier, orienté à l'est, avec une pente maximale approchant $25^{\circ}$, est parsemé de coulées dont le front pierreux atteint 0,5 $\mathrm{m}$ de hauteur. Le matériel en surface des coulées est généralement grossier et forme un pavage de blocs et de cailloux, des plages fines n'apparaissant que très localement. Ce versant possède une couverture neigeuse hivernale dont l'épaisseur dépasse un mètre et qui persiste partiellement au sommet jusqu'au mois de juillet sous la forme d'une corniche.

Le second versant étudié, au nord-ouest, est recouvert par de grosses coulées imbriquées avec des fronts atteignant $1,5 \mathrm{~m}$ de hauteur, sur une pente d'environ $30^{\circ}$. Contrairement à ce que l'on observe sur le versant opposé, les plages fines dominent en surface et les éléments grossiers se limitent aux fronts des coulées et à de larges stries. En raison de son exposition aux vents dominants, ce versant possède un couvert neigeux discontinu et relativement peu épais, permettant une pénétration plus profonde du gel dans le sol. L'existence

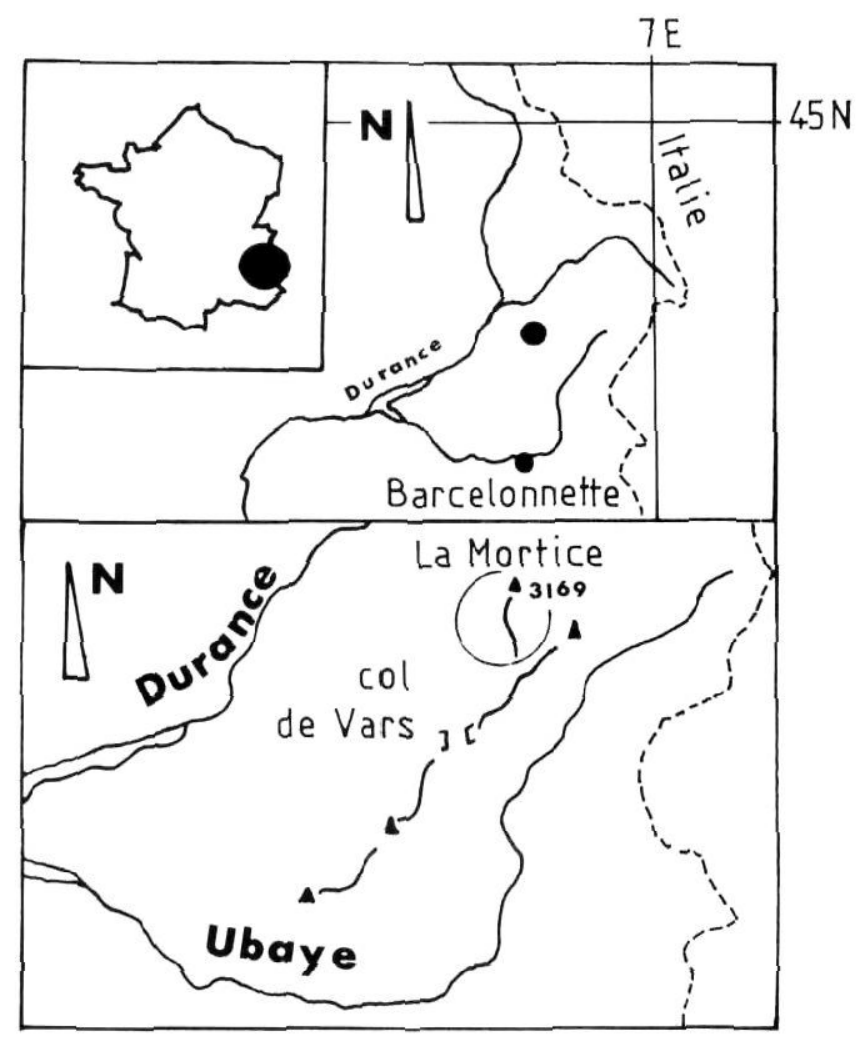

FIGURE 1. Situation géographique de La Mortice.

Location map of La Mortice. 
de coulées de solifluxion possédant un front de plusieurs mètres de hauteur laisse supposer la présence d'un pergélisol.

\section{RÉSULTATS}

\section{FRONTS PIERREUX ET LITAGE ASSOCIÉ SUR LE VERSANT EST}

\section{Description macroscopique de la stratification}

La coupe 1 (fig. 2 et 3) est réalisée dans une coulée dont la pente avoisine $25^{\circ}$, avec une épaisseur au niveau du front d'environ $40 \mathrm{~cm}$. La stratification, continue tout au long des cinq mètres de la tranchée, consiste en une alternance de niveaux légèrement ondulés riches en matrice, à éléments grossiers jointifs (support clastique), notamment dans la zone distale de la coulée, ou dispersés dans la masse fine (support matriciel), et de niveaux caillouteux à structure ouverte. L'épaisseur des lits est comprise entre 5 et $25 \mathrm{~cm}$. La limite supérieure des lits riches en matrice est nette, alors que leur limite inférieure est plus floue.

Les lits caillouteux ont une structure ouverte; cependant, les plus profondément enterrés tendent à être partiellement colmatés. Ils présentent des granoclassements verticaux généralement très nets, à polarité inverse dans le cas du pavage de surface et le plus fréquemment doubles dans les lits enterrés, avec une zone basale inverse et une zone sommitale normale. La disposition des éléments grossiers est fortement anisotrope. Ils sont imbriqués à la manière de tuiles, avec leur grand axe orienté dans le sens de la pente. En surface et au niveau du front, ces éléments sont dans leur majorité relevants par rapport à la pente générale de la coulée. Dans les lits enterrés, la disposition des cailloux suit les ondulations de la stratification.

\section{Granulométrie}

La matrice a une texture essentiellement sableuse ou sablo-silteuse, le pourcentage de silts étant compris entre 8,5 et $32 \%$, celui des argiles entre 6,5 et $16 \%$. La série de six prélèvements effectués dans un même lit riche en matrice montre une évolution nette d'amont en aval, marquée par un appauvrissement significatif en éléments inférieurs à 50 micromètres (fig. 4).

Sur une même verticale, on constate un enrichissement croissant en particules fines avec la profondeur, essentiellement en silts vers $40 \mathrm{~cm}$ et en argile silteuse vers $60 \mathrm{~cm}$.

\section{Microscopie}

Le lit riche en matrice de l'échantillon 1, prélevé dans la partie amont de la tranchée (fig. 2), possède une porosité élevée, estimée à environ $25 \%$. Elle est constituée par des vésicules arrondies ou mamelonnées (fig. 5), qui passent localement à de grands vides lamellaires. Les éléments grossiers, dont la taille dépasse $1 \mathrm{~cm}$, ont une disposition subparallèle au litage, alors que les petits graviers et les sables grossiers ont une orientation moins bien marquée. La fraction fine, quartzo-micacée, a une distribution porphyrique. On

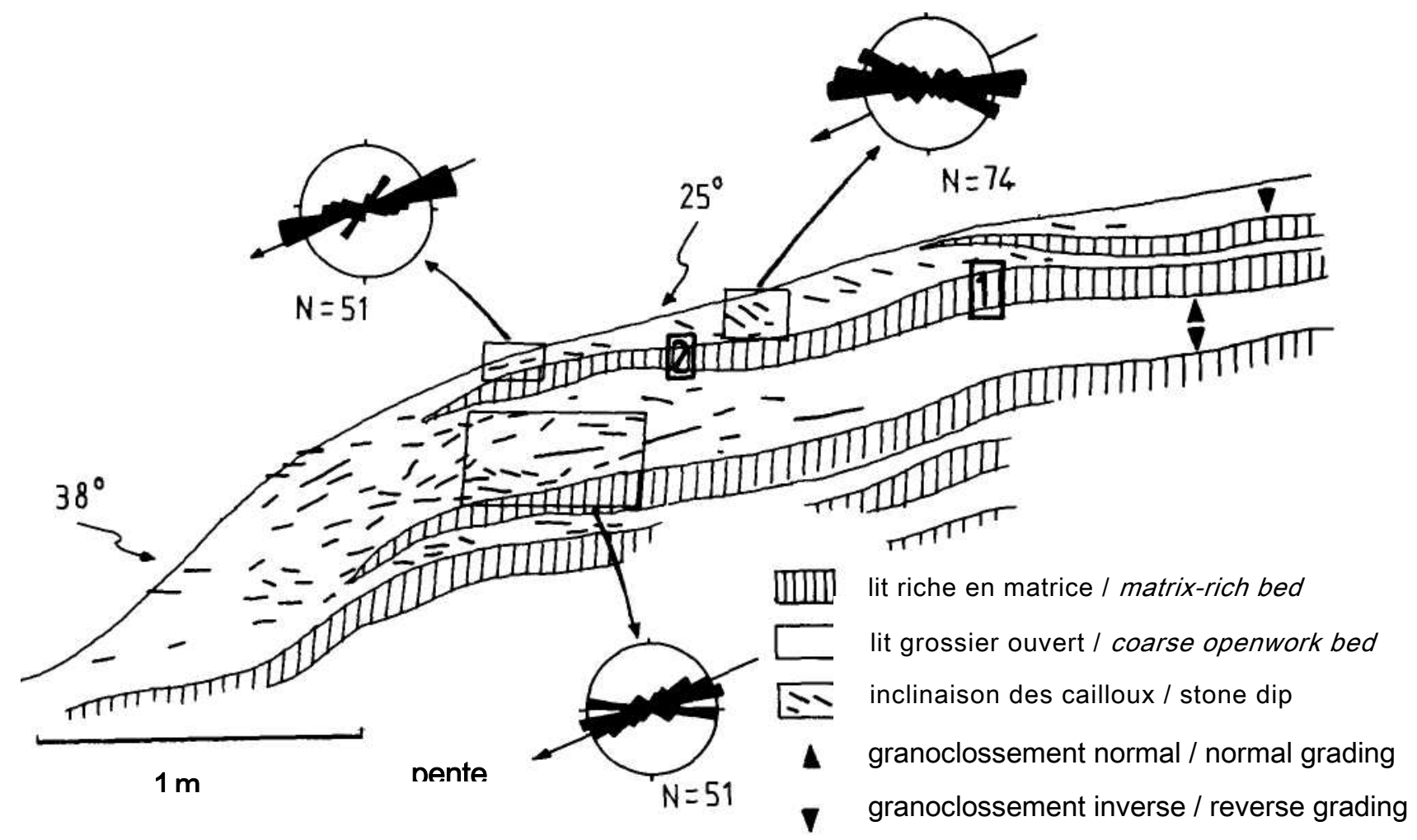

FIGURE 2. Schéma de la coupe 1, versant est. Sur les diagrammes d'inclinaison des cailloux, le cercle représente dix éléments. Les prélèvements pour l'analyse micromorphologique sont notés par des rectangles numérotés.

Sketch of section 1, east skie. The drcle on stone dip diagrams represents ten measurements. Numbeod rectangles indicate the position of samples for microscopie analys/s. 


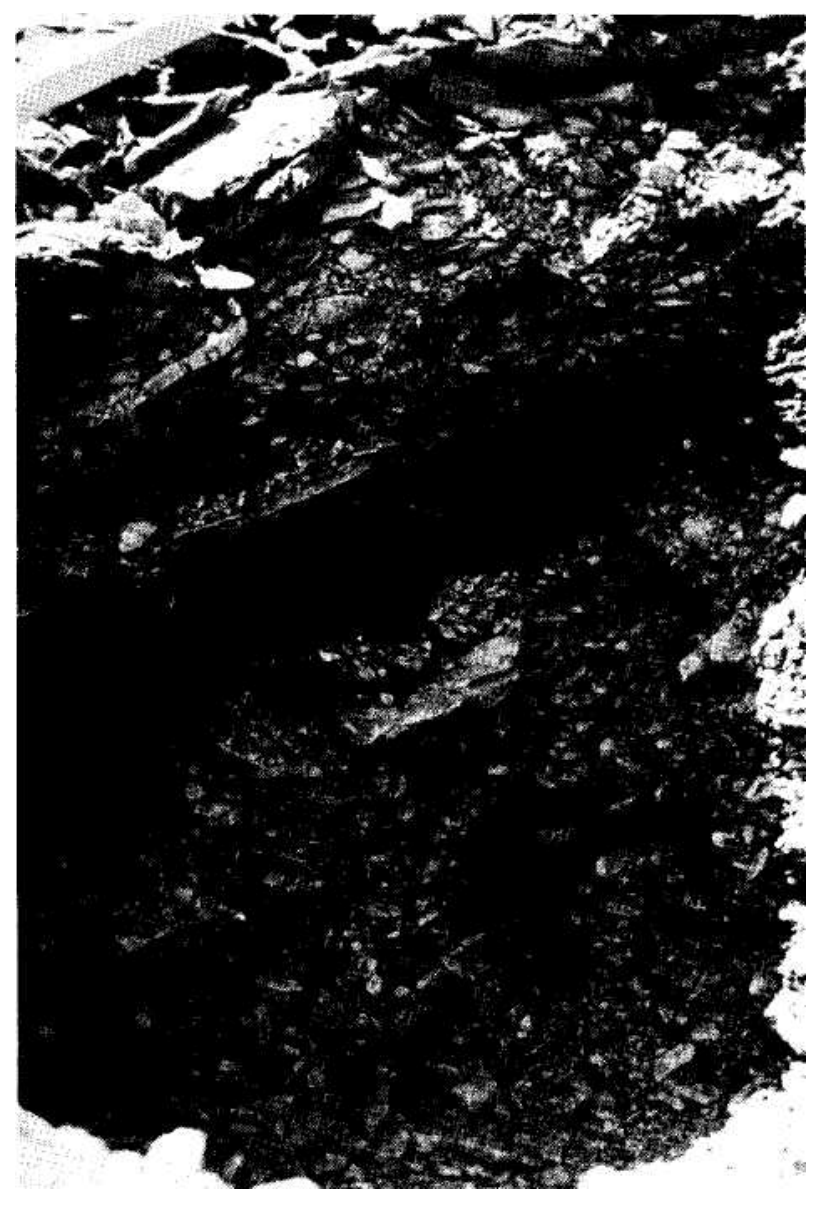

FIGURE 3. Détail de la coupe 1. Sous le pavage superficiel, alternent des lits riches en matrice et des lits grossiers ouverts.

Section 1, détail: alternating matrix-rich and coarse openwork beds under a superficiel stone pavement.

n'observe pas de traits texturaux bien différenciés du fond matriciel; néanmoins, la fraction de la taille des sables fins jusqu'aux argiles présente localement des plages litées, sous forme d'intercalations, de revêtements dans les cavités ou de ponts entre les graviers (fig. 6). L'ensemble du fond matriciel présente un caractère éluvial net.

Dans le lit grossier du même échantillon, la porosité (environ $40 \%$ ) est constituée de cavités d'entassement des graviers et des sables grossiers. La fraction argilo-silteuse est distribuée sous forme de coiffes sur la face supérieure des éléments grossiers. Ces coiffes sont fréquemment vésiculaires et possèdent une surface irrégulière; elles contiennent quelques éléments de la taille des sables grossiers et ne présentent pas de litage interne net.

Le second échantillon, situé à proximité du front de la coulée (fig. 2), présente des caractères intermédiaires entre le lit riche en matrice et le lit grossier de l'échantillon 1. Quelques plages sont relativement riches en matrice et ont une structure vésiculaire; le reste du fond matriciel est constitué par l'entassement de graviers et de sables grossiers avec une coiffe de fraction fine sur leur face supérieure. Dans ce dernier type de plages, apparaissent de nombreuses concentra-

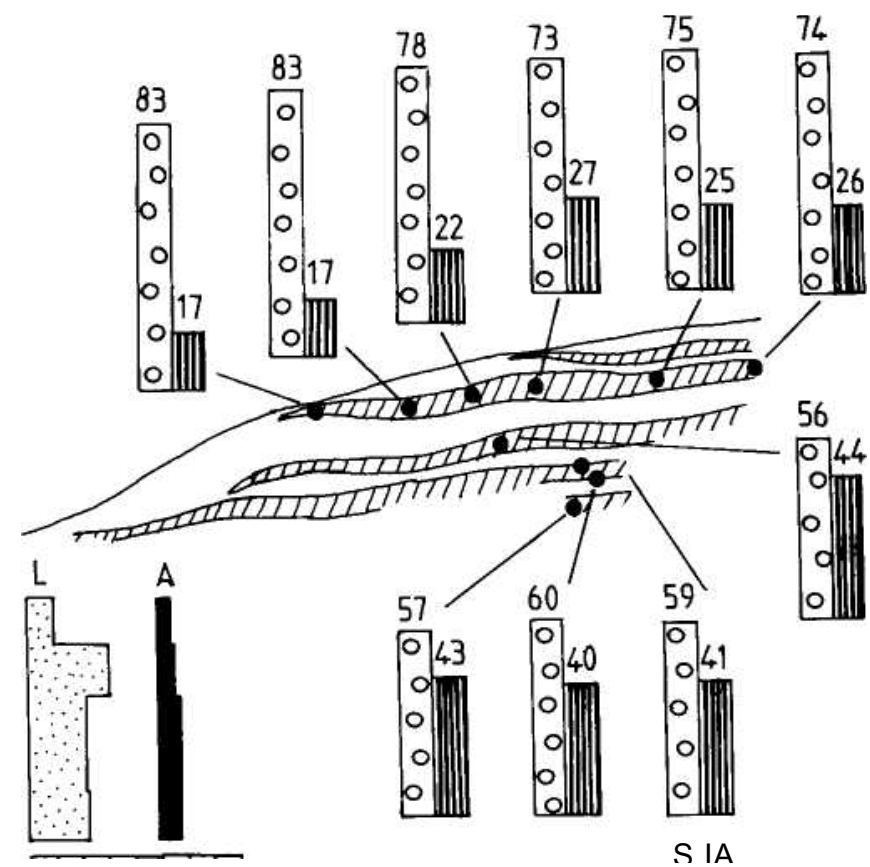

$020 \quad 400 \quad 20 \%$

FIGURE 4. Évolution longitudinale et verticale de la granulométrie dans la coupe 1. S: sable; LA: silt + argile. À gauche, diagramme montrant l'évolution verticale des proportions de silt (L) et d'argile (A).

Grain-size évolution along longitudinal and vertical axes in section 1. S: sand; LA: silt + clay. On the left, diagram showing vertical distribution of silt (L) and clay (A).

tions de sables fins sans matrice interstitielle, formant des entassements entre les éléments plus grossiers (fig. 7).

Variantes de la stratification

Les différentes tranchées réalisées sur le versant est ont permis de mettre en évidence une certaine variabilité dans les caractères de la stratification. Des coulées présentent ainsi des lits à structure ouverte minces, discontinus et avec une granulométrie beaucoup moins grossière que celle du front pierreux et du pavage (fig. 8). Par ailleurs, le redressement des éléments grossiers du front est parfois très accusé, certains cailloux étant disposés verticalement. Ce phénomène s'observe lorsque le lit riche en matrice situé sous la coulée perce le pavage en aval du front et forme un ostiole contre lequel bute la coulée.

\section{FRONTS PIERREUX ET SOLS STRIÉS ASSOCIÉS SUR LE VERSANT NORD-OUEST}

La coupe 2 (fig. 9) recoupe une épaisse coulée associée à un bourrelet de matériel fin en aval, à partir duquel partent latéralement des stries caillouteuses (fig. 10). La hauteur du front atteint $80 \mathrm{~cm}$. En coupe, on distingue un niveau pierreux ouvert superficiel dont l'épaisseur croît en direction du front, un épais niveau riche en matrice, contenant quelques passées de graviers ouvertes mais discontinues, un mince lit d'éléments grossiers ouvert, dont le pendage est d'abord parallèle à la pente puis relève vers la surface dans la zone du front. Cet ensemble repose sur un niveau colmaté, qui affleure en aval du front et forme un gros bourrelet en forme 


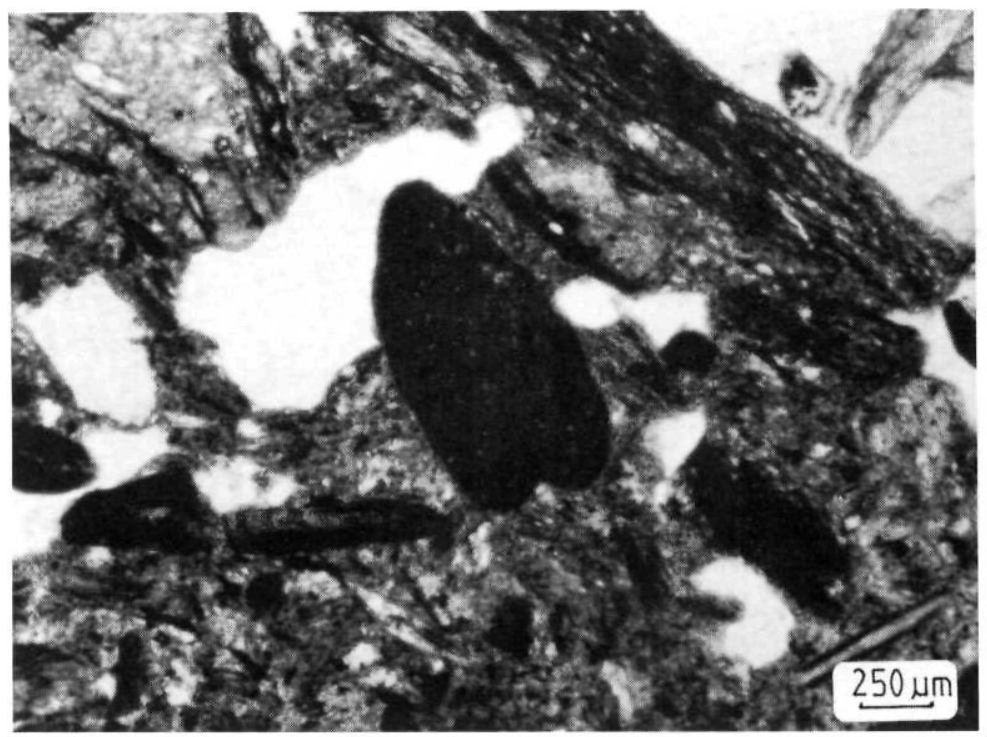

FIGURE 5. Organisation microscopique d'un lit riche en matrice (échantillon 1, coupe 1). La porosité est constituée de vésicules.

Microscopic organization of matrix-rich bed (sample 1, section 1). Porosity is vesicular.

de dôme, avec quelques fragments grossiers épars en surface.

La texture du lit riche en matrice est sablo-silteuse (sables: $65 \%$, silts: $21 \%$, argile: $14 \%$ ). En lames minces, son organisation est proche de celle du lit riche en matrice de la coupe 1, mais avec une structure lamellaire mieux développée.

\section{DISCUSSION}

L'analyse des différentes coupes réalisées dans les versants de La Mortice confirme le rôle des coulées de solifluxion à front pierreux dans le développement de la stratification des dépôts. Les mécanismes d'acquisition de cette stratification sont peu différents de ceux observés dans les Andes (Francou, 1988, 1990).

En surface et au niveau du front de la coulée, se produit une ségrégation des éléments grossiers sous l'action d'une cryoexpulsion très active et du ruissellement diffus. L'efficacité de l'expulsion en surface des éléments grossiers a pu être observée indirectement lors des mesures de déplacement des coulées à l'aide de portions de tubes enterrés. La majorité des tubes, d'une longueur de $15 \mathrm{~cm}$, enfoncés juste sous la surface étaient en effet remontés d'environ $3 \mathrm{~cm}$ quatre ans après leur implantation sur le site (J.-P. Coutard, comm. orale). Par ailleurs, une expérience en cours sur des cailloux marqués, d'environ $5 \mathrm{~cm}$ de diamètre et $2 \mathrm{~cm}$ d'épaisseur, enterrés dans une nappe fine affleurant sur le versant nord-ouest, a fourni les premiers résultats suivants: $7 \%$ des cailloux enfouis à $5 \mathrm{~cm}$ de profondeur et $3,5 \%$ de ceux enfouis à $10 \mathrm{~cm}$ ont été expulsés au bout d'une année.

On ne dispose pas de mesure sur le ruissellement, mais son activité est suggérée par l'abondance des microformes triées présentes sur le dos des coulées et celle, plus locale, de petits rills entamant les nappes fines.

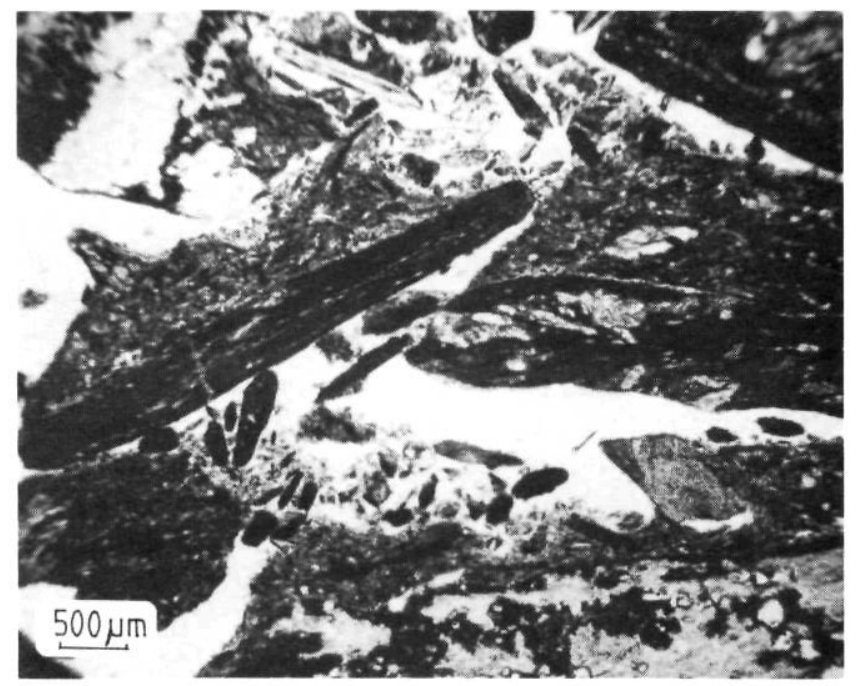

FIGURE 7. Organisation microscopique d'un lit riche en matrice à proximité du front de coulée (échantillon 2, coupe 1). Cet échantillon présente un très net appauvrissement en produits matriciels par rapport à celui dont proviennent les figures 5 et 6 . Noter les coiffes de matrice sur les fragments de schiste et les entassements de sables lavés entre ces éléments.

Microscopic organization of a matrix-rich bed close to the lobe front (sample 2, section 1). This sample presents a strong deficit of fine particles compared to Figures 5 and 6 . Note the matrix cappings of schist fragments and the loose sand infillings in between. 


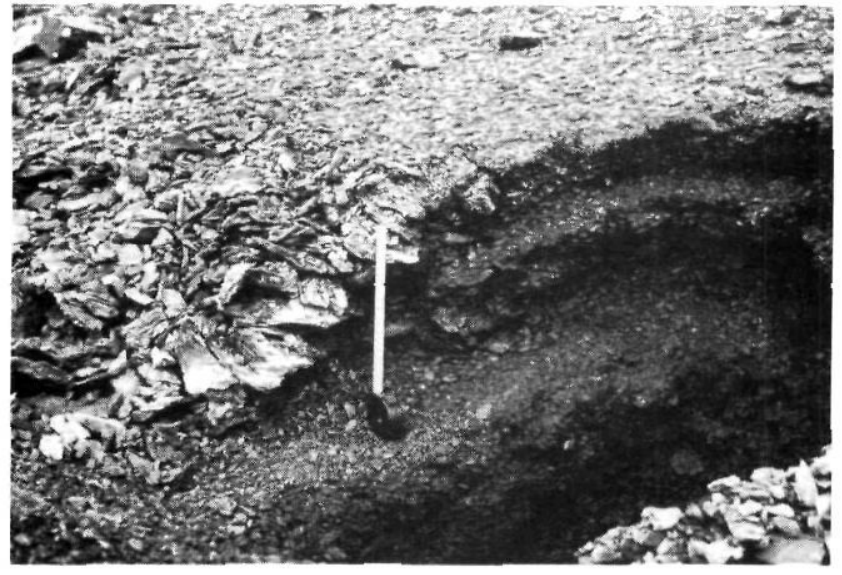

FIGURE 8. Coupe d'une coulée avec des lits grossiers ouverts peu developpés, contrairement au pavage de surface, versant est.

Stratified deposit with poorly developed operwork beds compared to the coarse surface pavement on the east side.

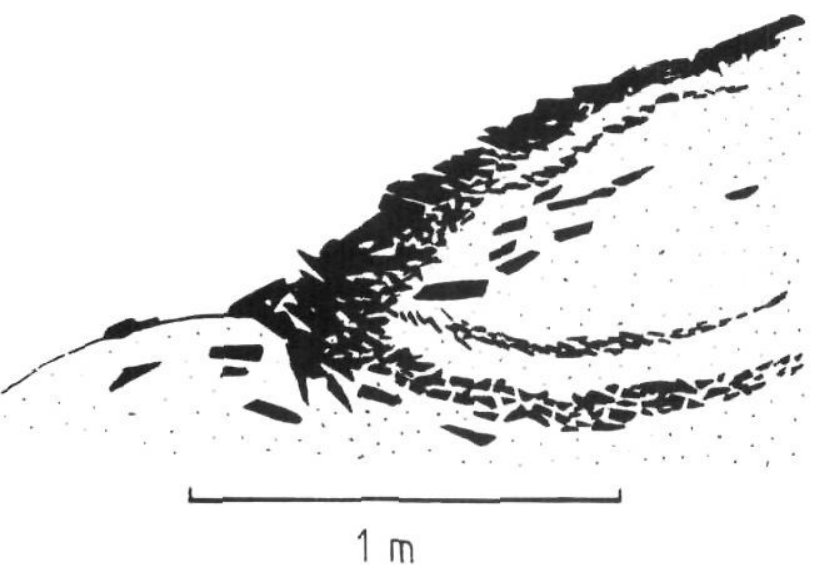

FIGURE 9. Schéma de la coupe 2, versant nord-ouest.

Sketch of section 2, northwest side.

Le pavage et le front pierreux ont tendance à acquérir un granoclassement inverse par "effet de crible", les éléments les plus fins migrant progressivement vers la base.

Le cœur de la coulée, appauvri en cailloux, forme un lit riche en matrice qui progresse par cryoreptation et ensevelit progressivement le front pierreux. Ces éléments sont incorporés au tapis sur lequel avance la coulée, permettant ainsi la constitution d'un lit grossier enterré à structure ouverte. Lorsque la coulée recouvre un pavage, le lit grossier présente généralement un granoclassement double. Par ailleurs, les cailloux, qui sont fortement relevants dans le pavage superficiel, prennent une inclinaison conforme à la stratification.

L'examen en lames minces des lits riches en matrice confirme le rôle majeur de la ségrégation de glace en lentilles, initiatrice de la cryoreptation, dans la dynamique de progression de la coulée (Coutard et al., 1988; Van Vliet-Lanoë, 1988a). Cependant, l'abondance des vésicules est typique de l'effondrement des agrégats au cours des phases de sursaturation en eau au dégel et marque une tendance à des mouvements plus rapides à l'état boueux, surimposés à la cryoreptation.

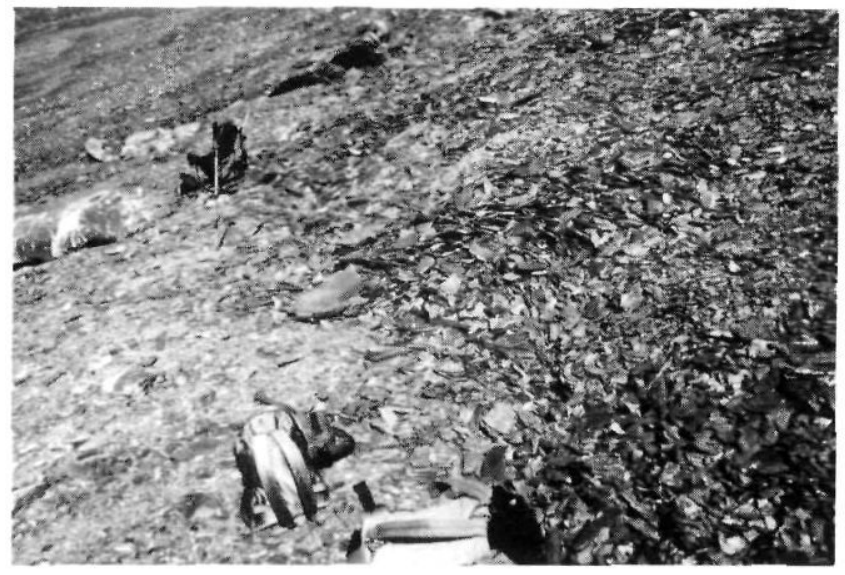

FIGURE 10. Coupe 2, versant nord-ouest. Fronts caillouteux d'épaisses coulées, dominant des extrusions de matériel fin. Ces bourrelets sont séparés par des stries caillouteuses, alimentées par les matériaux grossiers des fronts situés en amont.

Section 2, northwest side. Thick stony fronts with broad sorted pebbly stripes. The stripes composed of coarse material are fed by the overhanging fronts while the fine matrix forms ostioles.

Ces mouvements entrent dans le cadre de la gélifluxion et expliquent certaines vitesses relativement élevées, supérieures à 4 à $5 \mathrm{~cm}$.an-1, notées sur le versant est (Coutard et al., 1988).

Les analyses granulométriques et micromorphologiques montrent également l'importance des processus d'éluilluviation dans le fonctionnement des coulées. La coulée étudiée présente un appauvrissement en produits matriciels croissant en direction du front, corrélativement à un enrichissement des dépôts sous-jacents. On calcule que la composition de la matrice au niveau du front correspond à une perte d'environ $53 \%$ de silt et d'argile par rapport à la composition moyenne de la matrice sur l'ensemble du dépôt analysé. Vers $40 \mathrm{~cm}$ de profondeur, l'accumulation concerne surtout les particules silteuses, alors que les argiles sont exportées plus profondément. Cette recharge en silts des lits situés sous la coulée peut être rapprochée des "semelles limoneuses" décrites par Van Vliet-Lanoë (1988a, 1989) dans de nombreux dépôts périglaciaires soumis à une éluviation intense.

En lames minces, l'éluviation se traduit par l'apparition de figures de résidualisation du fond matriciel, en particulier au niveau du front. Dans le prélèvement amont, la matrice, appauvrie en argile de manière diffuse, reste abondante et emballe les sables grossiers et les graviers. À proximité du front de la coulée, cette organisation cède la place à un entassement libre d'éléments grossiers, avec des coiffes silteuses sur leur face supérieure et des concentrations résiduelles de sables fins dans les vides.

Dans l'ensemble, les dépôts lités de La Mortice présentent les traits sédimentologiques caractéristiques reconnus tant en milieu actif andin qu'en milieu hérité (grèzes litées): types de contacts entre les lits, avec des lits riches en matrice à limite supérieure nette et limite inférieure plus diffuse, granoclassement des lits grossiers à structure ouverte, fabrique imbriquée des cailloux avec le grand axe majoritairement orienté dans le sens de la pente et faiblement relevant. 
Toutefois, certaines différences apparaissent par rapport aux formations stratifiées étudiées dans les Andes:

1) la granulométrie des lits à structure ouverte est en général moins grossière que celle des éléments formant le front de la coulée;

2) les lits sont ondulés, alors que dans les Andes, la limite supérieure des lits riches en matrice est franchement tabulaire et rectiligne;

3) ces ondulations des lits riches en matrice s'accusent jusqu'à former des bourrelets qui perçent la surface en aval des fronts de coulées, lesquels présentent alors un fort redressement des éléments grossiers.

Ces différences sont particulièrement accusées sur le versant nord-ouest de La Mortice.

Les ondulations de la stratification et les ostioles perçant le pavage en aval des coulées résultent du gonflement différentiel des lits sous l'action du gel, phénomène déjà mis en évidence par Van Vliet-Lanoë (1987). Cet auteur décrit, après Herz et Andréas (1966), des déformations des dépôts de pente occasionnés par les différences de gélivité, qui peuvent aboutir à la formation de lits festonnés, voire à une pseudostratification. Par comparaison avec les formations andines, la cryoturbation est favorisée par une pénétration plus profonde du gel dans le sol, notamment sur le versant nord-ouest qui ne possède qu'une médiocre couverture neigeuse hivernale.

Les processus éluviaux, en accusant les contrastes de texture, contribuent également au développement des ostioles. En effet, l'accumulation de silts favorise la formation d'un niveau très gélif sous les coulées, qui est directement à l'origine des extrusions en aval. En revanche, la nappe de surface se résidualise et devient progressivement moins favorable à la ségrégation de glace en lentilles.

La présence des ostioles a pour conséquence un blocage partiel des coulées, dont le front présente alors un redressement généralisé des cailloux. Pour une part, ces éléments ne sont pas incorporés dans les lits grossiers, mais transitent en surface et alimentent les stries grossières ou le pavage mobile en aval des coulées.

La comparaison des dépôts lités de La Mortice et des Andes met donc clairement en évidence l'influence de certains paramètres sur la stratogénèse des dépôts de pente périglaciaires. Le développement de la stratification paraît dû à la présence de cycles gel-dégel superficiels fréquents, printaniers et automnaux dans les Alpes, sur l'ensemble de l'année dans les Andes, qui favorisent la cryoexpulsion des éléments grossiers et la constitution de pavages et de fronts pierreux. L'enfouissement régulier des éléments grossiers du front, qui forment ainsi un lit à structure ouverte, est perturbé dans les Alpes par la cryoturbation associée au cycle de geldégel profond saisonnier. Cette cryoturbation est d'autant plus active que les différences de texture entre les lits sont accentuées par une intense redistribution de la matrice (fig. 11).

Tout se passe comme si, parallèlement aux processus stratogéniques de type andin, se produisaient des transferts de matière hors du système constitué par la coulée, qui s'opposent au développement d'un litage régulier. Ces transferts correspondent à des départs de matrice par éluviation et à un transit en surface des éléments les plus grossiers du front vers le pavage ou les stries caillouteuses en aval, au lieu d'être ensevelis par l'avancée de la coulée.

On suppose que l'accentuation de ces phénomènes dans les milieux des hautes latitudes conduit à produire des dépôts avec des lits discontinus, voire sans stratification clairement exprimée. Ainsi, au Spitzberg, Van Vliet-Lanoë (1989) a montré l'importance de l'éluviation couplée à la cryoturbation dans la dynamique de versant. La matrice, lavée dans les niveaux superficiels, s'accumule au toit du pergélisol, créant de cette manière un gradient de gélivité négatif. Ce gradient est responsable de la formation d'injections silteuses évoluant en ostioles à la surface et ne pouvant être à l'origine que d'une stratification très passagère (Herz et Andréas, 1966).

Le type de stratification développé dans les dépôts de pente de La Mortice est très semblable à ceux décrits mais interprétés différemment par Soutadé (1975) dans les Pyrénées orientales et Vergès (1982) au mont Ventoux, dans le Vaucluse. Ces dépôts sont également associés en surface à des nappes-coulées à front pierreux, avec un pavage plus ou moins continu. Au mont Ventoux, ces formes partiellement couvertes par la végétation et dont l'altitude avoisine $1600 \mathrm{~m}$ seraient actuellement inactives.

II semble donc que les mécanismes mis en évidence à La Mortice soient caractéristiques du domaine périglaciaire des latitudes moyennes, à climat sub-océanique ou subméditerranéen.

\section{CONCLUSIONS}

Les observations réalisées à La Mortice mettent en évidence le développement d'une stratification dans les formations de pente périglaciaires, en relation avec une dynamique de coulées à front pierreux. Ces observations permettent d'étendre au domaine alpin, où le gélisol possède un

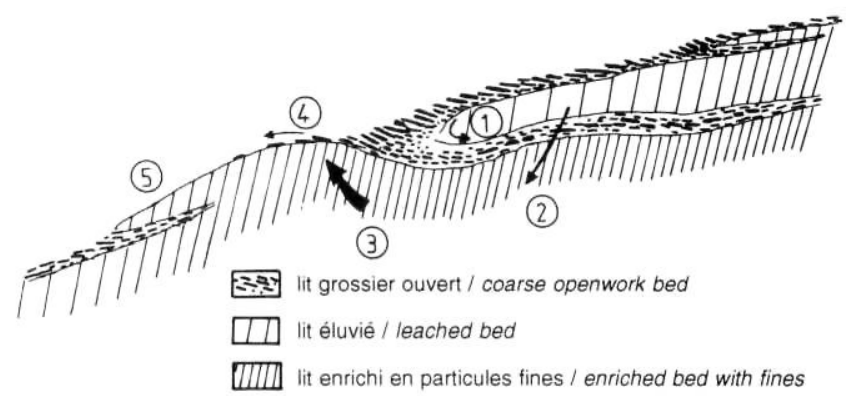

FIGURE 11. Schéma de fonctionnement d'une coulée: 1) progression de la coulée par cryoreptation et enfouissement des cailloux du front; 2) éluviation de la matrice; 3) extrusion du lit enrichi en particules fines; 4) transit en surface de l'ostiole d'une partie des éléments grossiers du front; 5) départ d'une coulée boueuse.

Sketch of the processes occurring within a sheet: 1) sheet advancing by frostcreep and burying of front stones; 2) translocation of fine particules; 3) formation of an ostiole; 4) transit of part of the front stones over the ostiole; 5) start of a mudflow. 
caractère essentiellement saisonnier, celles précédemment effectuées par Francou $(1988,1990)$ en milieu andin à cycles gel-dégel journaliers. Au delà d'une grande similitude de certaines figures sédimentologiques et microscopiques dans les dépôts des deux domaines, apparaissent un certain nombre de disparités liées aux différences des régimes thermiques et hydriques, concernant notamment la continuité et la régularité des lits.

Si l'acquisition de la stratification est bien liée à la présence de nombreux cycles gel-dégel superficiels, qui favorisent la ségrégation des éléments grossiers, sa physionomie semble très dépendante de la profondeur du gélisol saisonnier et de l'intensité du lavage subi par les coulées. La formation d'ostioles en aval des coulées, provoquée par un gel saisonnier profond et par les redistributions de matrice sous l'action des percolations, perturbe l'enfouissement régulier des fractions grossières triées au niveau des fronts et conduit au développement d'un litage ondulé et discontinu. L'accentuation de ces phénomènes dans les milieux des hautes latitudes permet d'expliquer la rareté des dépôts lités dans ces régions, à l'inverse des montagnes tropicales.

Pour mieux cerner l'influence des divers facteurs intervenant dans l'expression de la stratification des dépôts de pente, il serait souhaitable de répéter ce type d'analyse dans d'autres milieux ou dans des contextes lithologiques différents. En effet, l'influence de plusieurs autres processus, tels que les coulées boueuses (Wasson, 1979; Van Steijn et al., 1984; Gardner et al., 1991), les coulées sèches (Wasson, 1979), le glissement des débris sur des plaques de neige ou de verglas (Tricart et Cailleux, 1967; Van Steijn et al., 1984), les avalanches de neige et des apports de débris par le vent (Hétu, 1991) a été invoquée pour expliquer l'origine du litage de nombreux dépôts périglaciaires. Cependant, dans le contexte de La Mortice, ces processus semblent peu efficaces et incapables de produire les figures observées. Le domaine d'application du modèle stratogénique proposé ici reste donc à tester plus largement.

\section{REMERCIEMENTS}

Les auteurs tiennent à remercier J.-P. Coutard, J.-C. Ozouf et J.-P. Texier qui ont accepté de corriger les versions préliminaires de cet article, ainsi que les relecteurs de la revue, M. Allard, J. Gozdzik et J. Vandenberghe pour leur nombreuses suggestions.

\section{RÉFÉRENCES}

Bertran, P., Coutard, J.-P., Francou, B., Ozouf, J.-C. et Texier, J.-P., 1992. Données nouvelles sur l'origine du litage des grèzes. Implications paléoclimatiques. Géographie physique et Quaternaire, 46: 97-112.
Coutard, J.-P., Gabert, P. et Ozouf, J.-C., 1988. Étude du processus de cryoreptation en divers sites de la Haute-Ubaye (Alpes du Sud). Bulletin du Centre de Géomorphologie du C.N.R.S., Caen, 34: 9-28.

Francou, B., 1988. Éboulis stratifiés dans les Hautes Andes Centrales du Pérou. Zeitschrift für Geomorphologie N.F., 32, 1: 47-76.

1990. Stratification mechanisms in slope deposits in high subequatorial moutains. Permafrost and Periglacial Processes, 1: 249-263.

Gardner, T.W., Ritter, J.B., Shuman, C.A., Bell, J.C., Sasowsky, K.C. et Pinter, N., 1991. A periglacial stratified slope deposit in the Valley and Ridge province of central Pennsylvania, USA: sedimentology, stratigraphy and geomorphic evolution. Permafrost and Periglacial Processes, 2: 141-162.

Herz, K. et Andréas, G., 1966. Untersuchungen zur Morphologie der Periglazialen Auftauschicht im Kongsfjordgebiet (Westspitzbergen). Petersmanns Mittellungen, 110 (3): 90-198.

Hétu, B., 1991. Éboulis stratifiés près de Manche d'Épée (Péninsule de Gaspé, Québec). Zeitschrift für Geomorphologie, 35 (4): 439-432.

Malaurie, J., 1968. Thèmes de recherches géomorphologiques dans le NordOuest du Groënland. Mémoires et Documents, C.N.R.S., Paris, hors série, $481 \mathrm{p}$.

Soutadé, G., 1975. Coulées de blocailles et éboulis lités fọnctionnels (Terregalles) à la Coma de Finistrelles. Pyrénées méditerranéennes. Revue de Géomorphologie dynamique, I: 1-12.

Tricart, J. et Cailleux, A., 1967. Le modelé des régions périglaciaires. S.E.D.E.S., Paris, $512 \mathrm{p}$.

Van Steijn, H., Van Brederode, L. et Goedheer, J.C., 1984. Stratified slope deposits of grèzes-litées type in the Ardèche region in south of France. Geografiska Annaler, 66A: 295-305.

Van Vliet-Lanoë, B., 1987. Cryoreptation, gélifluxion et coulées boueuses: une dynamique continue en relation avec le drainage et la stabilité de l'agrégation cryogénique, p. 203-226. In M. Pecsi et H.M French, édit., Loess and Periglacial Phenomena. Akadémial Kiado, Budapest: 203-226.

- 1988a. Approche morphologique et micromorphologique de la dynamique de solifluxion dans les massifs de la Font Sancte et du Chambeyron. Bulletin du Centre de Géomorphologie du C.N.R.S., Caen, 34: 29-45.

1988b. Teneur en glace et comportement thermique des sols cryoturbés. Leurs conséquences pour le développement des contraintes mécaniques dans les sols. Exemples pris au Spitzberg et dans les Alpes. Bulletin du Centre de Géomorphologie du C.N.R.S., Caen, 34: 119-135.

- 1989. Aspects dynamiques de versants de gélifraction en milieu arctique. Exemples du Kongsfjord, Spitzberg du Nord-Ouest. Notes et Comptes-rendus du groupe de travail « Régionalisation du Périglaciaire" , Comité national français de Géographie, fascicule XIV: 75-82.

Van Vliet-Lanoë, B. et Francou, B., 1988. Étude micromorphologique et dynamique comparative de sols striés et autres petites formes fluantes superficielles en milieu arctique, alpin et andin. Bulletin du Centre de Géomorphologie du C.N.R.S., Caen, 34: 47-63.

Vergès, V., 1982. Contribution à l'analyse et à la représentation cartographique des formations pédologiques en moyenne montagne calcaire. Thèse de $3^{\circ}$ cycle, Université de Paris VII, $235 \mathrm{p}$.

Wasson, R.J., 1979. Stratified debris deposits in the Hindu Kush, Pakistan, Zeitschrift für Geomorphologie N.F., 23(3): 301-320. 